\title{
Obtención de Cordierita por Sol-Gel a partir de fuentes nacionales cubanas.
}

\author{
P. PACHECO, A.N. HERNÁNDEZ, A. VERA, A. VALDÉS, E. OROPESA \\ Centro de Investigaciones para la Industria Minero Metalúrgica (CIPIMM - Cuba)
}

Para la síntesis de la Cordierita por el método de Sol-Gel se utilizó, el silicato de sodio, obtenido de la disolución de un residuo sólido proveniente de la producción de sulfato de aluminio a partir de caolín. Los precursores de magnesio y aluminio fueron obtenidos utilizando las tecnologías previamente desarrolladas por el CIPIMM. Los materiales fueron mezclados en un orden y cantidad determinada hasta obtener el Gel con estequiometría de cordierita. El gel fue lavado, secado, briqueteado y tratado térmicamente a diferentes temperaturas, lográndose cordierita en fase mayoritaria a los $1200^{\circ} \mathrm{C}$, lo cual fue corroborado mediante el análisis por difracción de rayos $\mathrm{X}$ de los productos del tratamiento térmico. El método utilizado comparado con los tradicionales permitió reducir la temperatura de cristalización, obtener productos más densificados y tecnología limpia.

Palabras claves: cordierita, sol-gel, sinterización, residuos.

\section{Cordierite obtention through sol-gel basing on cuban national sources}

For cordierite synthesis through the Sol-Gel method, the sodium silicate obtained from the dissolution of a solid residue coming from the aluminum sulfate production based on kaolin, was used. The sources of magnesium and aluminum were obtained using the technologies previously developed by CIPIMM. The elements were mixed in a determined order and quantity to obtain a Gel with cordierite stechiometry. The gel was washed and dried and briquettes were made to treat them at different temperatures, obtaining cordierite in a major phase at $1200^{\circ} \mathrm{C}$. This was checked through the X-Ray diffraction analysis of the products coming from the thermical treatment. The method used when compared to the traditional ones, permitted to reduce crystallization temperature, obtaining more dense products and clean technology.

Key words: cordierite, sol-gel, syntherization, residues.

\section{INTRODUCCIÓN.}

La cordierita es un sistema cristalino ortorrómbico, forma cristales prismáticos cortos y seudohexagonales. La fórmula global más aceptada de la cordierita es $2 \mathrm{MgO} \cdot 2 \mathrm{Al}_{2} \mathrm{O}_{3} \cdot 5 \mathrm{SiO}_{2}$, con una composición estequiométrica de 51,36 \% de $\mathrm{SiO}_{2}$; $34,86 \%$ de $\mathrm{Al}_{2} \mathrm{O}_{3}$ y $13,78 \%$ de $\mathrm{MgO}$ (1). Son materiales ampliamente utilizados debido a sus características especiales, como baja expansión térmica, constante dieléctrica, densidad, que permite su aplicación con ventajas sobre materiales cerámicos tradicionales o aleaciones metálicas, por ejemplo como sustrato o empaquetamientos electrónicos, circuitos integrados de microondas para uso en comunicaciones por radar, pigmentos, etc.

Los pigmentos cerámicos del tipo cordierita presentan la resistencia a la acción de las altas temperaturas y los medios agresivos. La valoración visual del color de estos pigmentos es: azul, azul celeste, celeste, azul oscuro, cuando se sustituye el Mg por Co; el Ni por Mg da colores grisáceos, verdosos, verde azulado, celeste - verdoso; cuando se sustituye el Al por Cr se obtienen colores rosados, con tinte grisáceo; Cuando se introduce $\mathrm{Mn}$ por $\mathrm{Mg}$ el color va de rosado a beige, con tinte castaño. $(2,3,4,5$ y 7$)$

El principal problema de los sistemas basados en cordierita reside en la dificultad de obtener un material densificado antes de la cristalización de la cordierita durante el procesa- miento térmico. En este sentido, la obtención de un precursor de cordierita de alta homogeneidad por una ruta no convencional, tal como Sol-Gel, evita la necesidad de altos gastos energéticos, lo que resulta altamente ventajoso.

El método de preparación Sol-Gel puede clasificarse en dos formas: en la primera la sílice se incorpora en forma de suspensión coloidal, y el resto de los componentes se incorpora en forma de sales disueltas; la mezcla homogénea o sol se transforma en gel por desestabilización. Posteriormente se hace que pierda agua y densifique. En la segunda forma todos los componentes o partes de ellos se introducen en forma de compuestos metalorgánicos (alcóxidos). Tras la reacción de hidrólisis de los alcóxidos se produce la policondensación que origina la gelificación del sol.

Los materiales utilizados usualmente en cuerpos de cordierita obtenidos mediante procesos Sol-Gel son: como fuente de magnesio y aluminio los nitratos o sulfatos, y como fuente de silicio el tetraetilortosilicato (TEOS), el silitrane (TEASI - egH) y el metabisilicato de sodio. (6)

En el trabajo se pretende, a partir de fuentes nacionales precursoras de $\mathrm{MgO}, \mathrm{Al}_{2} \mathrm{O}_{3}$ y $\mathrm{SiO}_{2}$ obtener geles con estequiometría similar a la de cordierita, evidenciando la evolución de las fases cristalinas con el aumento de la temperatura de retención, durante la sinterización de cordierita. 


\section{PARTE EXPERIMENTAL.}

\subsection{Materiales utilizados.}

Se utilizaron pulpas de hidróxido de magnesio, soluciones de sulfato de aluminio y silicato de sodio. Estos productos fueron obtenidos al nivel de laboratorio a partir de fuentes nacionales.

\subsubsection{OBTENCIÓN DE SILICATO DE SODIO.}

El silicato de sodio se obtuvo a partir de un residuo de caolín que resulta de la lixiviación ácida del caolín para obtener sulfato de aluminio.

Las características del residuo sólido del caolín son: 74,21 $\%$ de $\mathrm{SiO}_{2} ; 14,69 \%$ de $\mathrm{Al}_{2} \mathrm{O}_{3} ; 0,069 \%$ de $\mathrm{MgO}$ y $1,43 \% \mathrm{Fe}_{2} \mathrm{O}_{3}$; con una granulometría menor de $0,02 \mathrm{~mm}$ y el silicio que contiene tiene características amorfas.

El silicato de sodio se obtuvo disolviendo el silicio del residuo de caolín utilizando hidróxido de sodio a una concentración de $100 \mathrm{~g} / \mathrm{L}$, con una relación de 13,3 Líquido/Sólido, temperatura de $60{ }^{\circ} \mathrm{C}$, y tiempo de agitación de 20 minutos.

\subsubsection{MATERIAS PRIMAS CON CONTENIDO DE AL2O3 Y $\mathrm{DE} \mathrm{MgO}$.}

Como fuente principal de óxido de aluminio se utilizó sulfato de aluminio en solución, obtenido de la lixiviación ácida del caolín con ácido sulfúrico y como fuente de magnesio se utilizó hidróxido de magnesio en pulpa, obtenido de la precipitación del magnesio contenido en salmueras residuales utilizando hidróxido de amonio.

\subsection{Análisis químico de los materiales utilizados.}

Se realizó análisis de $\mathrm{SiO}_{2}, \mathrm{Al}_{2} \mathrm{O}_{3}$ y $\mathrm{MgO}$ a los materiales utilizados para conocer el aporte de óxidos de cada fuente individual, con el objetivo de formular la mezcla del gel con estequiometría similar a la cordierita.

\subsection{Obtención del gel.}

El gel se obtuvo a partir de pulpa de hidróxido de magnesio, solución de sulfato de aluminio y silicato de sodio.

Las adiciones para la formación del gel, se calcularon sobre la base del aporte de $\mathrm{SiO}_{2}, \mathrm{Al}_{2} \mathrm{O}_{3}$ y $\mathrm{MgO}$ que contiene cada fuente individual (solución de silicato de sodio, solución de sulfato de aluminio y pulpa de hidróxido de magnesio).

Los cálculos para el mezclado se realizaron con el propósito de obtener unos $50 \mathrm{~g}$ de sólido en el gel.

Para formular las mezclas definimos que:

$\mathrm{m}=$ número de óxidos en cada material utilizado: $\mathrm{MgO}$; $\mathrm{Al}_{2} \mathrm{O}_{3} ; \mathrm{SiO}_{2} .(\mathrm{m}=3)$

$\mathrm{n}=$ número de materiales utilizados: $\mathrm{Mg}(\mathrm{OH})_{2} ; \mathrm{Al}_{2} \mathrm{SO}_{4^{\prime}}$; $\mathrm{Na}_{2} \mathrm{SiO}_{3}$. $(\mathrm{n}=3)$ terial j $\mathrm{b}_{\mathrm{i}}=$ número máximo de gramos necesarios del óxido $\mathrm{i}$; para $\mathrm{MgO}=13,78$; para $\mathrm{Al}_{2} \mathrm{O}_{3}=34,86$; y para $\mathrm{SiO}_{2}=51,36$.

$\mathrm{x}_{\mathrm{j}}=$ número de gramos necesarios del material $\mathrm{j}$ que deberá mezclase. $\left(x_{i}>0\right)$

La cantidad total del óxido i contenido en todos los compuestos mezclados está dada por:

$$
a_{i 1} x_{1}+a_{i 2} x_{2}+a_{i 3} x_{3}+\cdots+a_{i n} x_{n}
$$

Puesto que esta cantidad total deberá ser igual al número máximo necesario del óxido i, entonces se resuelve el siguiente sistema de ecuaciones

$$
\begin{aligned}
& a_{11} x_{1}+a_{12} x_{2}+a_{13} x_{3}=b_{1} \\
& a_{21} x_{1}+a_{22} x_{2}+a_{23} x_{3}=b_{2} \\
& a_{31} x_{1}+a_{32} x_{2}+a_{33} x_{3}=b_{3}
\end{aligned}
$$

Durante el lavado de las primeras pruebas se determinó pérdidas de $\mathrm{MgO}$ de un $60 \%$, por lo que se adicionó un exceso de pulpa de hidróxido de magnesio equivalente a las pérdidas de $\mathrm{MgO}$.

Para obtener el gel, primeramente se mezcló la pulpa de hidróxido de magnesio con la solución de sulfato de aluminio, hasta obtener una mezcla. Homogénea a pH 4. Después se le adicionó la solución de silicato de sodio y se obtuvo un gel muy homogéneo a $\mathrm{pH} 8,4$, de color blanco opaco. En total se adicionó $266 \mathrm{~mL}$ de solución de sulfato de aluminio, $76 \mathrm{~g}$ de Pulpa de hidróxido de magnesio y $580 \mathrm{~mL}$ solución de silicato de sodio. En la figura I se puede observar un esquema del proceso de mezclado para obtener el gel.

\subsection{Obtención de las briquetas.}

Las briquetas se formaron prensando $10 \mathrm{~g}$ de gel lavado y seco (humedad de $48 \%$ ) a una presión de 100 bar en una prensa hidráulica de fabricación rusa tipo P10. En total se formaron 4 briquetas.

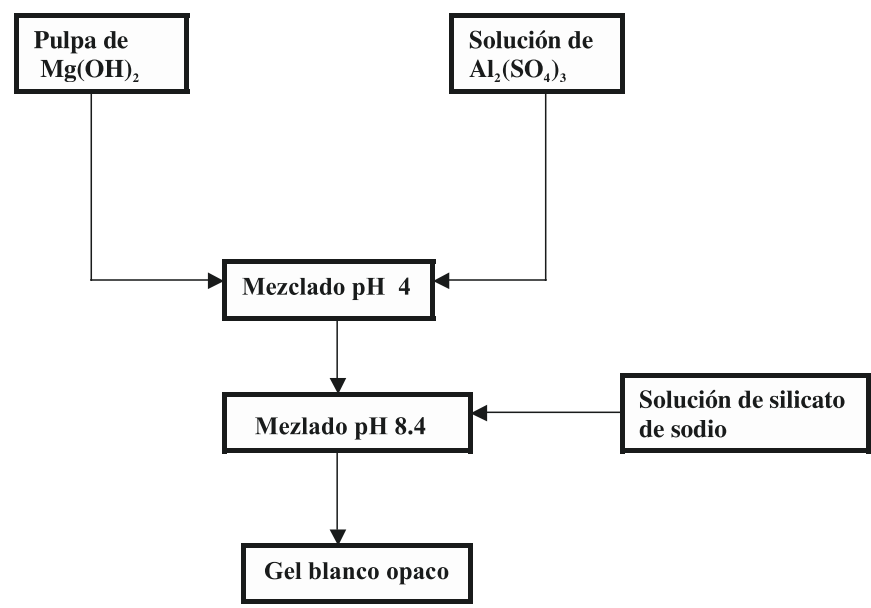

Figura I. Esquema del proceso de mezclado para formar el gel. 


\subsection{Tratamiento térmico.}

Las briquetas con estequiometría similar a la de cordierita se sometieron a idénticos tratamientos térmicos pero a diferentes temperaturas de retención (a temperaturas 1000, 1100, $1150,1200{ }^{\circ} \mathrm{C}$ ) donde permanecieron dos horas. La velocidad de calentamiento fue de $10{ }^{\circ} \mathrm{C}$ por minuto desde $28{ }^{\circ} \mathrm{C}$ hasta la temperatura de retención. Estos tratamientos fueron disenados para observar la evolución de fases cristalizadas en las distintas temperaturas.

Los tratamientos térmicos se llevaron a cabo en un horno mufla de laboratorio, con control de calentamiento programable hasta temperaturas de $1600^{\circ} \mathrm{C}$ de la firma inglesa LHENTON THERMAL FURNACE LTA.

\subsection{Técnica de caracterización.}

Se realizaron análisis químico de $\mathrm{SiO}_{2}, \mathrm{Al}_{2} \mathrm{O}_{3}$ y $\mathrm{MgO}$ a los materiales utilizados para formar el gel y al gel lavado y seco, utilizando el método de análisis de espectrometría con Plasma Inductivamente Acoplado (SPECTROFLAME EDP), de la firma alemana Spectro Analytical Instrument.

Para seguir la secuencia de cristalización de las diferentes muestras se utilizó la Difracción de Rayos X, empleando un Difractómetro PHILIPS PW 1710 con radiación K $\alpha$ del Fe y filtro de $\mathrm{Mn}$, en el intervalo de $6^{\circ}$ a $70^{\circ}$ en $2 \theta$.

Además, se realizó la caracterización estructural de las briquetas tratadas a 1150 y $1200{ }^{\circ} \mathrm{C}$, empleando microscopio óptico polarizado JENAPOL con cámara Sony acoplada.

\section{RESULTADO Y DISCUSIÓN.}

\subsection{Resultados del análisis de $\mathrm{SiO}_{2}, \mathrm{AL}_{2} \mathrm{O}_{3}$ y $\mathrm{MgO}$ a los ma- teriales utilizados.}

La solución de silicato de sodio obtenida es transparente y tiene un contenido de $44 \mathrm{~g} / \mathrm{L}$ de $\mathrm{SiO}_{2}, 2,41 \mathrm{~g} / \mathrm{L}$ de $\mathrm{Al}_{2} \mathrm{O}_{3} \mathrm{y}$ menos de $4,15 \times 10^{-4} \mathrm{~g} / \mathrm{L}$ de $\mathrm{MgO}$. Esto representa el $79 \%$ de extracción de $\mathrm{SiO}_{2}$, dado que el residuo sólido remanente de la disolución de la sílice, está constituido por mica y cuarzo (poco soluble) lo que justifica el \% de extracción.

La concentración de $44 \mathrm{~g} / \mathrm{L}$ de silicio fue la adecuada para su conservación, pues a concentraciones mayores del $5 \%$ en solución, precipita en forma de gel.

La solución de sulfato de aluminio obtenida contiene: 60,1 $\mathrm{g} / \mathrm{L}$ de $\mathrm{Al}_{2} \mathrm{O}_{3}, 0,23 \mathrm{~g} / \mathrm{L}$ de $\mathrm{SiO}_{2}$ y 0,014 g/L de MgO.

La composición del hidróxido de magnesio seco contenido en la pulpa es la siguiente: $61,5 \%$ de $\mathrm{MgO}, 0,06 \%$ de $\mathrm{Al}_{2} \mathrm{O}_{3^{\prime}}$ $0,22 \%$ de $\mathrm{SiO}_{2}$ con $76,4 \%$ de humedad.

Las características, volumen y peso de los materiales utilizados en el mezclado se presentan en la tabla I.

\subsection{Formación de gel precursor con estequiometría de cor- dierita.}

El gel obtenido presentó características de composición estequiométrica similares a la cordierita. En la Tabla II se presentan los resultados de las características del gel y el cálculo de la relación, entre los contenidos de $\mathrm{SiO}_{2}, \mathrm{Al}_{2} \mathrm{O}_{3}$ y $\mathrm{MgO}$ en el gel.
TABLA I. CARACTERÍSTICAS DE LOS MATERIALES UTILIZADOS.

\begin{tabular}{|l|c|c|c|c|}
\hline \multirow{2}{*}{$\begin{array}{c}\text { Denominación } \\
\text { del material }\end{array}$} & \multicolumn{3}{|c|}{ Concentración } & $\begin{array}{c}\text { Volumen } \\
\text { o Peso } \\
\text { utilizado }\end{array}$ \\
\cline { 2 - 5 } & $\mathrm{SiO}_{2}$ & $\mathrm{Al}_{2} \mathrm{O}_{3}$ & $\mathbf{M g O}$ & $580 \mathrm{~mL}$ \\
\hline $\begin{array}{l}\text { Solución de silicato de } \\
\text { sodio }\end{array}$ & $44,1 \mathrm{~g} / \mathrm{L}$ & $2,41 \mathrm{~g} / \mathrm{L}$ & $<4,15 \times 10^{-4} \mathrm{~g} / \mathrm{L}$ & $266 \mathrm{~mL}$ \\
\hline $\begin{array}{l}\text { Solución de sulfato de } \\
\text { aluminio }\end{array}$ & $0,23 \mathrm{~g} / \mathrm{L}$ & $60,1 \mathrm{~g} / \mathrm{L}$ & $0,014 \mathrm{~g} / \mathrm{L}$ & $76 \mathrm{~g}$ \\
\hline $\begin{array}{l}\text { Pulpa de hidróxido de } \\
\text { magnesio }\end{array}$ & $0,22 \% *$ & $0,60 \% *$ & $61,05 \% *$ & $\%$ \\
\hline
\end{tabular}

$\left({ }^{*}\right)$ - Análisis en base seca.

TABLA II. CARACTERÍSTICAS DEL GEL LAVADO Y SECO.

\begin{tabular}{|l|c|c|c|}
\cline { 2 - 4 } \multicolumn{1}{c|}{} & \multicolumn{3}{c|}{ Composición en \% } \\
\cline { 2 - 4 } \multicolumn{1}{c|}{} & $\mathrm{SiO}_{2}$ & $\mathrm{Al}_{2} \mathrm{O}_{3}$ & $\mathbf{M g O}$ \\
\hline Análisis químico del gel. & 34,76 & 24,97 & 10,82 \\
\hline Relación entre $\mathrm{SiO}_{2}, \mathrm{Al}_{2} \mathrm{O}_{3}$ y MgO & 49,27 & 35,39 & 15,34 \\
\hline Estequiométrica de cordierita. & 51,36 & 34,86 & 13,78 \\
\hline
\end{tabular}

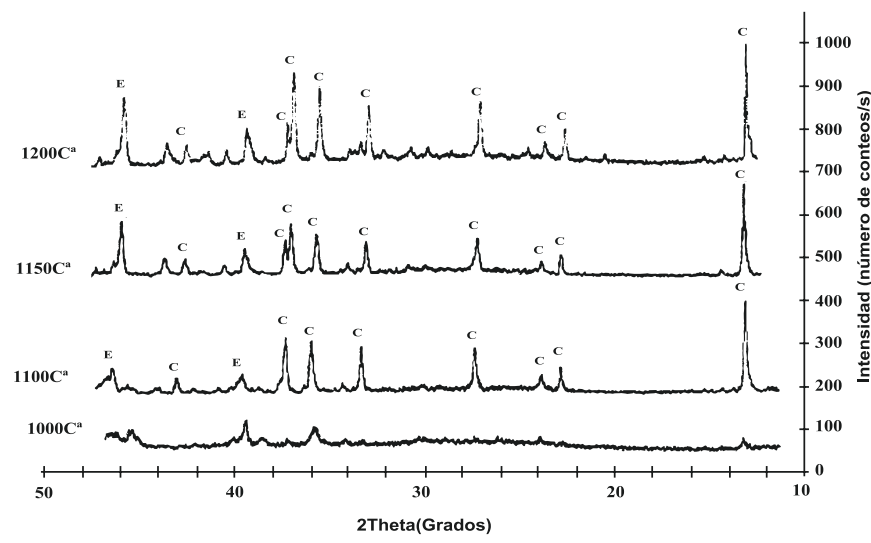

Figura II. Secuencia de cristalización de las muestras en el tratamiento térmico. C - Cordierita, E - Espínela

\subsection{Sinterización de cordierita.}

Las briquetas tratadas térmicamente (temperaturas 1000, $1100,1150,1200{ }^{\circ} \mathrm{C}$ ) fueron analizadas por difracción de rayos $\mathrm{X}$ dando como resultado, a partir de $1100{ }^{\circ} \mathrm{C}$, la presencia de cordierita. Esto es observable por el máximo de difracción que aparece a la distancia interplanar de $8,5 \mathrm{~A}^{\circ}$ de la fase - Cordierita

Se obtuvieron los mejores resultados a la temperatura de $1200{ }^{\circ} \mathrm{C}$ (ver figura II), donde las fases detectadas fueron cordierita como fase mayoritaria y espínela como fase secundaria y el resto amorfo.

Se realizó análisis por microscopía óptica a las briquetas tratadas a $1150,1200{ }^{\circ} \mathrm{C}$, las imágenes se muestran en las figuras III y IV.

\section{CONCLUSIONES}

A partir de materias primas nacionales es posible la obtención de sólidos con estequiometría de cordierita mediante técnicas de Sol-Gel.

Fue realizable la síntesis de cordierita donde las fases detectadas fueron cordierita como fase mayoritaria y espínela como fase secundaria y el resto amorfo, y los mejores resultados fueron a la temperatura de $1200^{\circ} \mathrm{C}$. 


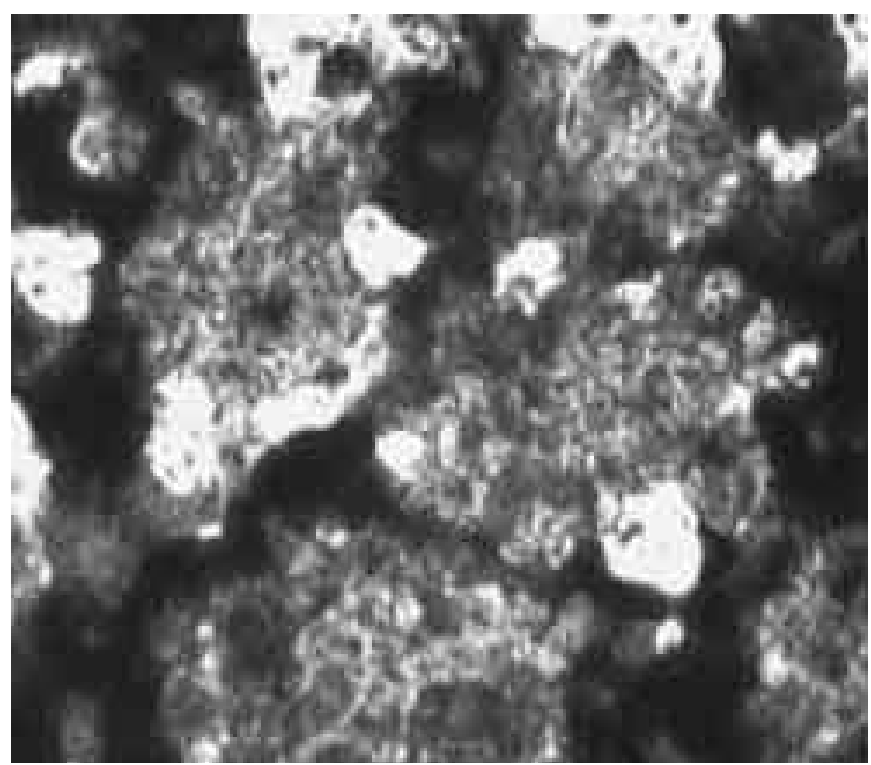

a) SD.- Cordierita $1200^{\circ} \mathrm{C}$, Nicoles / / 200

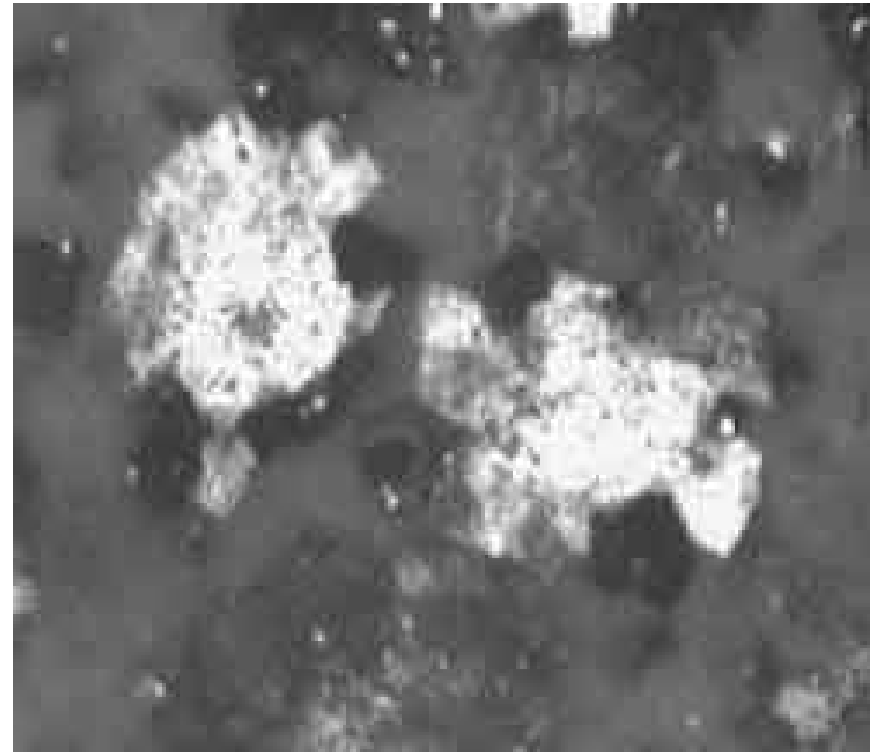

b) SD.- La misma del lado pero con Nicoles X 200

Figura III. Imagen de la sección pulida de la cordierita sintetizada por sol-gel a $1200^{\circ} \mathrm{C}$

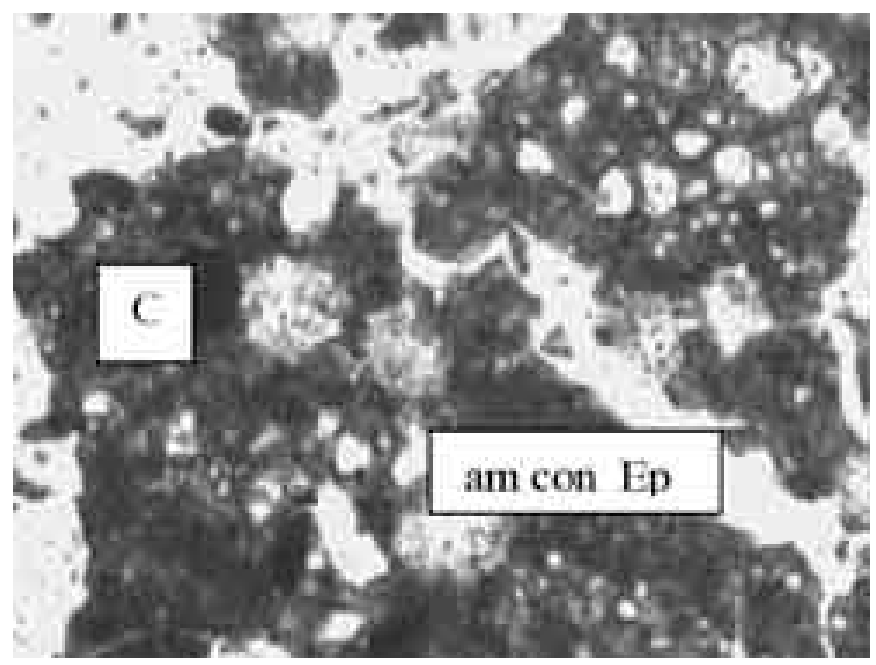

a) SD.- Cordierita $1150{ }^{\circ} \mathrm{C}$; Agregado de Cordierita color pardo verduzco con amígdalas ( am ), redondeadas rellenas de espínela ( Ep ). Nicoles // 200

Figura IV. Imagen de la cordierita sintetizada por sol-gel a $1150^{\circ} \mathrm{C}$

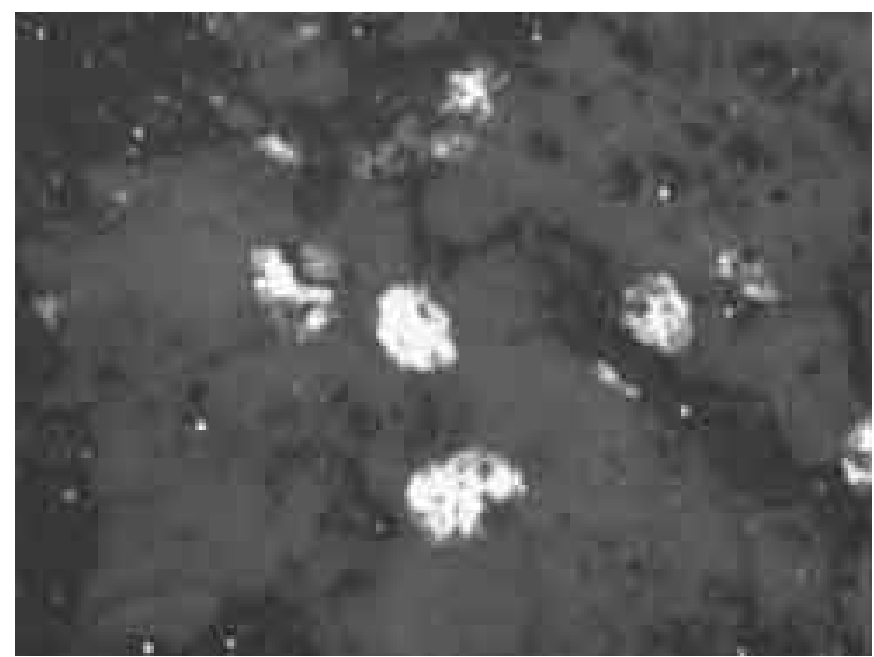

b) SD.- La misma del lado pero con Nicoles $X 200^{x}$

\section{BIBLIOGRAFÍA}

1. Edward Henry Kraus," Mineralogía", 5ta ed., Revolucionaria, 1970.

2. Cherepanina L.I., Maiganecsoderzhazhkie pigmenty cordierito bodo tipa, Steklo i keramika, 1985.

3. Cherepanina L.I., Sintez i issledovanie keramicheskikh pigmentov sistemy $\mathrm{MgO}-\mathrm{NiO}-\mathrm{Al}_{2} \mathrm{O}_{3}-\mathrm{SiO}_{2}$, Steklo i keramika, 1979

4. Cherepanina L.I., Keramicheskie pigmenty kordierito tipa sitemy $\mathrm{MgO}$ - $\mathrm{CoO}-\mathrm{Al}_{2} \mathrm{O}_{3}-\mathrm{SiO}_{2}$, Steklo i keramika, 1978.

5. V.P. Tyrkov, L.I.Cherepanina. "Keramicheskie pigmenty tipa kordierita". Steklo i keramika, 5, 22-23, 1981.

6. J. Vila, M. C. Muñoz, C. Valentín, M. Sales y J. Alarcón. “Cristalización de cordierita a partir de geles y vidrios estequiométricos. Estudio comparativo". Bol. Soc. Esp. Cerámica y Vidrio, Vol. 38, No. 1, 21- 28, 1999.

7. G.N. Maslennikova, N.P. Felina. "Keramicheskie pigmenty tipa kordierita". Steklo i keramika, 9, 18-19, 1987.

8. M. Raigón. “Síntesis y propiedades de cordierita. Influencia del tratamiento

mecánico de las materias primas empleadas". Bol. Soc. Esp. Cerámica y Vidrio, Vol. 38, No.1, 54, 1999.

9. J. Vila, C. Valentín, M. C. Muñoz, M. Sales y J. Alarcón. “Cristalización de cordierita en vidrios derivados del sistema cuaternario $\mathrm{CaO}-\mathrm{MgO}-\mathrm{Al}_{2} \mathrm{O}_{3}$ $\mathrm{SiO}_{2}$ ". "Influencia de la composición del vidrio". Bol. Soc. Esp. Cerámica y Vidrio, Vol. 37, No. 5, 390, 1998.

10. B. Nekrasov. “El silicio". 341-355. “Química general”. Ed. MIR.1969.

11. M.E. Tyrrell, G.V. Gebbs y H.R. Ghell. "Síntesis de Cordierita". Vol. 594 Burean of Mines.

12. "Preparación de silicato de sodio a partir de arenas mexicanas". Bol. Soc. Esp. Cerámica y Vidrio, Vol. 31, No. 2, 131, 1992.

Recibido: 09.12.01

Aceptado: 11.08 .02 